\title{
Hemodialysis Catheters in Infants: A Retrospective Single-Center Cohort Study
}

\author{
Ma, Grace M Y ; Ventura, Luís Marcelo ; Amiribadi, Afsaneh ; Gnannt, Ralph ; Nemec, Rose ; Noone, \\ Damien ; Licht, Christoph ; Amaral, Joao ; Temple, Michael ; Muthasami, Prakash ; Parra, Dimitri ; \\ Chamlati, Racha ; Connolly, Bairbre
}

\begin{abstract}
PURPOSE Evaluate technical aspects and outcomes of insertion/maintenance of hemodialysis (HD) central venous catheter (CVC) during infancy. MATERIALS AND METHODS Single-center retrospective study of 29 infants who underwent 49 HD-CVC insertions between 2002 and 2016. Demographics, procedural, and post-procedural details, interventional radiology (IR) maintenance procedures, technical modifications, complications, and outcomes were evaluated. Technical adjustments during HDCVC placement to adapt catheter length to patient size were labeled "modifications." CVCs requiring return visit to IR were called IR-maintenance procedures. Mean age and weight at HD-CVC insertion were 117 days and $4.9 \mathrm{~kg}$. RESULTS Of the 29 patients, 13 (45\%) required renal-replacement-therapy (RRT) as neonates, 10 (34\%) commenced RRT with peritoneal dialysis (PD), and 19 (66\%) with HD. Fifteen nontunneled and 34 tunneled HD-CVCs were inserted while patients were 1 year. Technical modifications were required placing 25/49 (51\%) HD-CVCs: 5/15 (33\%) nontunneled and 20/34 (59\%) tunneled catheters $(\mathrm{P}=.08)$. Patients underwent 6 dialysis-cycles/patient during infancy (mean 2.3), and a mean of 4.1 and 49 HD-sessions/catheter for nontunneled and tunneled HD-CVCs, respectively. Mean primary and secondary device service, and total access site intervals for tunneled HD-CVCs were 75, 115, and 201 days, respectively. A total of 26 of 49 (53\%) patients required IR-maintenance procedures. Nontunneled lines had greater catheter-related bloodstream infections per 1,000 catheter-days than tunneled HD-CVCs (9.25 vs. 0.85/1,000 catheter days; $\mathrm{P}=.02)$. Nineteen patients $(65 \%)$ survived over 1 year. At final evaluation (December 2017): 8/19 survived transplantation, 5/19 remained on RRT, 2/19 completely recovered, $1 / 19$ lost to follow-up, and 3 died at 1.3, 2, and 10 years. CONCLUSIONS Placement/maintenance of HD-CVCs in infants pose specific challenges, requiring insertion modifications, and IR-maintenance procedures to maintain function.
\end{abstract}

DOI: https://doi.org/10.1016/j.jvir.2020.01.020

Posted at the Zurich Open Repository and Archive, University of Zurich

ZORA URL: https://doi.org/10.5167/uzh-187941

Journal Article

Accepted Version

Originally published at:

Ma, Grace M Y; Ventura, Luís Marcelo; Amiribadi, Afsaneh; Gnannt, Ralph; Nemec, Rose; Noone, Damien; Licht, Christoph; Amaral, Joao; Temple, Michael; Muthasami, Prakash; Parra, Dimitri; Chamlati, Racha; Connolly, Bairbre (2020). Hemodialysis Catheters in Infants: A Retrospective Single-Center Cohort Study. Journal of Vascular and Interventional Radiology, 31(5):778-786. 
DOI: https://doi.org/10.1016/j.jvir.2020.01.020 


\title{
Hemodialysis catheters in infants - a retrospective single center cohort study
}

\begin{abstract}
Purpose: Evaluate technical aspects and outcomes of insertion/maintenance of hemodialysis(HD) central venous catheter(CVC) during infancy.

Materials and Methods: Single center retrospective study of 29 infants who underwent 49 HD-CVC insertions between 2002-2016. Demographics, procedural- and post-procedural details, IR-maintenance procedures, technical modifications, complications and outcomes were evaluated. Technical adjustments during HD-CVC placement to adapt catheter length to patient size were labeled 'modifications'. CVCs requiring return visit to IR were called IR-maintenance procedures. Mean age and weight at HD-CVC insertion were 117 days and $4.9 \mathrm{~kg}$.
\end{abstract}

Results: Of the 29 patients, 13(45\%) required renal-replacement-therapy (RRT) as neonates; 10(34\%) commenced RRT with peritoneal dialysis (PD) and 19(66\%) with HD. Fifteen non-tunneled and 34 tunneled HD-CVCs were inserted while $\leq 1$ year. Technical modifications were required placing 25/49(51\%) HD-CVCs: 5/15(33\%) non-tunneled and 20/34(59\%) tunneled catheters $(p=0.08)$. Patients underwent $\leq 6$ dialysis-cycles/patient during infancy (mean 2.3), and a mean of 4.1 and 49 HD-sessions/catheter for non-tunneled and tunneled HD-CVCs, respectively. Mean primary and secondary device service, and total access site intervals for tunneled HD-CVCs were 75, 115, and 201 days, respectively. 26/49(53\%) required IR-maintenance procedures. Non-tunneled lines had greater catheterrelated-bloodstream-infections per 1,000 catheter-days than tunneled HD-CVCs (9.25 vs $0.85 / 1,000$ catheter days; $p=0.02)$. Nineteen patients $(65 \%)$ survived over 1 year. At final 
evaluation (December 2017): 8/19 survived transplantation, 5/19 remained on RRT, 2/19 completely recovered, 1/19 lost to follow-up and 3 died at 1.3,2, and 10 years. Conclusion: Placement/maintenance of HD-CVCs in infants pose specific challenges, requiring insertion modifications, and IR-maintenance procedures to maintain function. 


\section{Introduction}

Children between $0-24$ months represent $1-2 \%$ of the pediatric population with end-stage renal disease (ESRD) (1). They require renal replacement therapy (RRT) or renal transplantation to survive (1). Transplantation offers the best prognosis, but is not performed until children reach 2-years/10 kg (1-3). RRT options include peritoneal dialysis (PD) and hemodialysis (HD). PD is preferred in infants given its improved preservation of residual renal function, fewer dietary restrictions, possible home dialysis and avoidance of central venous catheters (CVC) (4-9). Consequently, $84 \%$ of children $<2$ years requiring RRT receive PD (4-6). However, HD is necessary when PD is contraindicated or fails (7). In infants, $\mathrm{HD}$ is achieved through CVCs, as arteriovenous fistulae in children $<10 \mathrm{~kg}$ are problematic (8). Infants may require switches between RRT modes (PD/HD) and devices. HD-CVC placement in children with metabolic diseases requiring RRT share similar challenges as those with ESRD. HD in neonates and infants is challenging and reports from an interventional radiology (IR) perspective are scarce $(4,5,9-12)$.

The purpose of this study was to evaluate HD-CVC insertion and maintenance in infants. The hypothesis was that HD-CVC insertion/maintenance in infants would pose specific challenges due to patient size and limitations stemming from manufactured equipment.

\section{Materials and Methods}

\section{Study design and patient population}

Research Ethics Board approved retrospective, single-center, consecutive cohort analysis was performed of infants who underwent HD-CVC placement by IR at a tertiary level pediatric hospital between 2002 and 2016. Inclusion criteria were patients $\leq 1$ year of age at 
first HD-CVC insertion (tunneled or non-tunneled). Catheters not placed by IR (e.g. ICU, surgical team), or placed for reasons other than RRT (e.g. stem cell harvest, apheresis) were excluded. Catheter related adverse events and dialysis sessions were assessed through the first year of life, and overall patient outcomes were followed through the end of the study period (December 2017) or death.

\section{Data collection}

Data sources included electronic medical records, picture archiving and communication system (PACS; Centricity; GE Healthcare, Chicago, IL), in-house dialysis and IR databases. Data elements included: a) demographics (diagnosis, gender, age, weight at HDCVC insertion); b) procedural details (type and size of catheter, vein accessed, technical success, insertion challenges/modifications, complications); c) post-procedural details up to 1 year of age (number of dialysis cycles and HD sessions, primary and secondary device service intervals, total access site service interval, catheter IR-maintenance procedures, adverse events (AE)); and d) status at final review (alive, transplanted, RRT).

\section{Definitions}

An infant is a child $\leq 1$ year and a neonate is $\leq 6$ weeks of age. A "dialysis cycle" is the continuous period from beginning of one dialysis mode to the end of that mode (e.g. switching between HD and PD, renal transplantation, recovery, death) (12). A "hemodialysis session" was each period on hemodialysis within a day. HD-CVCs were categorized as non-tunneled (non-cuffed) or tunneled (cuffed), and as upper or lower central venous system based on vascular territory (catheter's venous pathway from entry site to tip). Target position for upper venous system was right atrium (RA), and for lower 
venous system was the inferior vena cava (IVC) (13-15). Technical adjustments during HD-CVC placement to adapt catheter length to patient size were labeled 'modifications'. Procedures requiring a return visit to IR (e.g. catheter exchanges, contrast evaluations, cuff adjustments, re-suturing) were called IR-maintenance procedures. Technical success, device service intervals, access site intervals and complication categorization were defined using SIR guidelines (16-19). Catheter-related infections were analyzed if they met SIR definitions of catheter-related sepsis/bacteremia, with no alternative source of infection, and resulted in catheter removal (16). Adverse events (AE) were classified as early (<30 days) or late (>30 days) with severity defined per SIR classification (Mild, Moderate, Severe, Life-threatening/Disabling, Death) (20). HD-CVC associated symptomatic thrombosis diagnosed by US were analyzed. Routine thrombosis screening was not performed per institutional practice.

\section{Technique and catheters}

The most appropriate HD-CVC (size; non-tunneled/tunneled; upper/lower venous system) was determined pre-procedure by a vascular access nurse, nephrologist and interventional radiologist, based on required flows, clinical stability and anticipated HD duration.

Prophylactic antibiotics were not used. Patients were placed in Trendelenberg position for upper venous system HD-CVCs $(13,21)$. Internal jugular and femoral veins were preferred access sites. Imaging (US, fluoroscopy) and Seldinger technique were employed (21). Small volume contrast hand injection was used to verify catheter tip and right atrium (RA) position per institutional practice. Catheters were heparin-locked (10 units/kg heparin, maximum 100 units/lumen) and secured in place. Heparin-lock post dialysis was based on 
patient weight and catheter volume (heparin 1,000 units/ml at 50units/kg, diluted to catheter fill volume).

\section{Patient characteristics}

Twenty-nine patients met inclusion criteria. They underwent 49 HD-CVCs at $<1$ year of age. Their diagnoses, age and mode of initial RRT are summarized in Table 1. Mean age and weight of infants with metabolic disease did not differ from ESRD patients $(p=0.16)$.

\section{Statistical analysis}

Microsoft Excel 2010 (Microsoft Corp, Redmond, Washington) and SPSS statistical software (version 23.0, IBM, Armonk, NY) were used. Categorical data was described with frequency distributions, percentages and compared using two-sided Chi-square tests. Two sample Student's t-test compared continuous variables. Significance was set at $p<0.05$.

\section{Results}

\section{Initiating RRT}

Thirteen (45\%) patients required RRT as neonates and 24(83\%) patients at less than 6 months (Table 1). One-third commenced RRT using PD, two-thirds with HD, with no significant age difference $(p=0.82)$.

\section{Procedural}

Forty-nine HD-CVCs were inserted in 29 patients at <1 year. US and fluoroscopy were used in 47/49 (96\%) placements; US only was used in 2/49 (4\%) (non-tunneled HD-CVCs placed in intensive care unit (ICU)). Size and type of HD-CVCs are shown in Table 2. In 
11(38\%) patients their first HD-CVC was non-tunneled (Table 1). There was no difference in ages or weights at insertion of non-tunneled versus tunneled HD-CVCs $(p>0.54)$.

Fifteen HD-CVCs (31\%) were non-tunneled: 5 upper and 10 lower venous systems. Those in the upper venous system were too long for the infants' thorax. Modifications for RA tip positioning was achieved by: (1) suturing catheter wings to the scalp behind the ear cephalad to insertion site $(n=4)$; (2) suturing to scalp plus placing catheter tip at the IVC/RA, with ability to withdraw the catheter into the SVC/RA if needed ( $n=1 ; 6.5$ French, $10 \mathrm{~cm}$ long HD-CVC). Without modifications, the 10 lower venous system HD-CVCs reached the iliac vein $(n=1)$, lower 1/3 IVC $(n=5)$, middle $1 / 3$ IVC $(n=3)$ and upper IVC $(\mathrm{n}=1)$. Thirty-four HD-CVCs were tunneled (all in the upper venous system). 20/34(59\%) required intra-procedural modifications to achieve RA position: (1) venous tip trimmed to reduce the distance between staggered ends $(n=17) ;(2)$ tip trimming, plus catheter pullback with burying the cuff in a caudal extension to the tunnel ("T-incision") $(n=3)$ (Table 3 and Figure 1).

With these modifications, technical success rates were $94 \%$ overall, $94 \%$ (32/34) for tunneled HD-CVCs and 93\%(14/15) for non-tunneled HD-CVCs. Technical failures, based on tip position/function, occurred in 2 tunneled and 1 non-tunneled HD-CVC (Table 3).

\section{Post-procedure (Table 3)}

Twenty-nine infants underwent 66 dialysis cycles (mean: 2.3 cycles/patient, range: 1-6), and a total of 1612 HD-sessions (mean: 4.1 HD-sessions/non-tunneled catheter and 49 sessions/tunneled catheter) (Table 3). Comparing non-tunneled to tunneled HD-CVCs, 
there were significant differences in mean number of sessions/catheter $(p=0.002)$, dwell times $(p=0.004)$, primary device service interval $(p=0.002)$ and total access site interval $(p<0.001)$

\section{IR-maintenance procedures}

Twenty-six (53\%) catheters (25 tunneled; 1 non-tunneled) in 13 patients required one or more catheter IR-maintenance procedures: 25 contrast evaluations, $6 \mathrm{HD}-\mathrm{CVC}$ re-suturing, and 1 non-tunneled catheter 'pulled back' to shorten it. Thirteen catheters required exchanges due to fibrin sheaths $(n=3)$, catheter fractures $(n=2)$, malposition/dislodgement $(n=7)$, and one exchanged empirically for dysfunction (function improved post-exchange). 11/13(85\%) were tunneled HD-CVC over-the-wire-exchanges and 2/13(15\%) were nontunneled to tunneled HD-CVCs exchanges using a new site. There was no significant difference in the IR-maintenance rates/1,000 catheter days for non-tunneled and tunneled HD-CVCs $(p=0.454)$ (Table 3).

\section{Catheter related adverse events}

There were 6 intra-procedural AEs (early AEs). Three patients had prolonged bleeding at the site: self-limited with pressure in 2 (SIR Mild) and persistent in 1 coagulopathic patient, ultimately requiring line removal (SIR Moderate). Two patients required a second puncture (SIR Mild). One patient experienced transient profound hypotension (etiology unclear) shortly after HD-CVC insertion, which responded to calcium chloride and phenylephrine without sequelae (SIR Mild). 
In four patients, 5 CRBSI occurred amongst 49 HD-CVCs (4 tunneled jugular, 1 nontunneled femoral), resulting in catheter removal. Overall infection rate was 1.03 infections/1,000 catheter-days, significantly lower among tunneled catheters compared to non-tunneled catheters ( 0.85 vs 9.25 per 1,000 catheter-days; $p<0.02)$. In 6 instances of laboratory-documented contaminants, HD-CVCs were not removed.

US diagnosed thrombosis in 10/29(34\%) symptomatic patients, associated with 12 catheters: 8 jugular venous thromboses with 3 extending into the SVC/brachiocephalic veins; 4 femoro-iliac venous thrombosis with 1 extending into the IVC. 8 were occlusive and 4 were non-occlusive. Four additional 'silent' venous occlusions were identified during HD-CVC insertions, (2 at <1 year; 2 at $>1$ year age). Two patients with upper venous system thromboses developed chylothoraces, at 5 and 10 days post HD-CVC insertion (Early SIR, Moderate); the latter patient suffered severe late sequelae (SIR Death) (Table 4). Trimming of the catheter was not associated with increased fibrin sheath/thrombosis ( $p>0.422)$. Incidence of thromboses ranged between $22-33 \%$ among all catheter sizes (Table 4). One patient had synchronous concomitant HD-CVC related infection and thrombosis. All tunnel extensions healed, but were associated with more cuff exposures than non-extended tunnels $(p=0.011)$.

\section{Outcomes}

There were 10 deaths in infancy: 9 confirmed, and one presumed (3 month old discharged for palliative care). None were catheter or CRBSI related, although 2 had unrelated sepsis (Table 5). Nineteen patients survived beyond infancy, with a mean follow-up of 5.9 years. At final review, 8/19(53\%) survived following transplantation (kidney $n=4$; liver $n=3$, 
multi-organ $n=1), 2 / 19(12 \%)$ remained on $\mathrm{HD}, 3 / 19(17 \%)$ on $\mathrm{PD}, 1 / 19(6 \%)$ fully recovered renal function and 2/19(12\%) lost to follow-up after clinical improvement. There were 3 late deaths: at 16 months (renal failure), 2 years (chylothorax, plastic bronchitis, vascular stenting) and 10 years (complications post multi-organ transplant).

\section{Discussion}

Although infant hemodialysis is now considered mainstream treatment, this study outlines IR challenges of HD-CVC insertion and management in patients <1 year of age (23-27). Comparative mortality rates for different RRT modes are difficult to ascertain because outcomes are determined by birth weight/gestation, ESRD etiology, pulmonary hypoplasia, residual renal function, family circumstances, with 5-fold increases in mortality in infants with neurological disorders and 4-fold if commencing RRT < 1year compared to > 1year (4, 28, 29). European guidelines (2014) suggest treatment initiation should consider predicted quality of life for patients/families, accessible resources, and prognosis for future renal transplantation (30). Initiation of infant dialysis bears important ethical dimensions, requiring multidisciplinary patient-specific decisions $(23,28,31,32)$. This study reflects some of these challenges of HD-CVC insertion and maintenance in infancy.

Reported mortality rates for ESRD in young children range between 18-50\%, depending on age and weight of cohorts (4, 5, 8-11). A $30 \%$ mortality has been reported in children $<10 \mathrm{~kg}$ on dialysis (4). In the current study, 10 (34\%) died in infancy despite RRT. The high proportion of neonates at RRT initiation (45\%) highlights their disease severity and associated co-morbidities. Of 19 survivors, 9 were successfully bridged to transplantation with multiple RRT cycles and nutritional support through a gastrostomy/gastrojejunostomy. 
The rate of renal transplantation $(28 \%)$ is towards the lower range in the pediatric literature of $28-89 \%(4,5,8-12)$, reflecting this infant population and consistent with rates among neonatal cohorts of $22 \%(23,27,32)$.

Vascular occlusion is a major concern. The rate of 1.9 symptomatic thromboses $/ 1,000$ catheter-days among tunneled HD-CVCs is consistent with literature (33). Asymptomatic thrombosis rates as high as $24.7 / 1000$ catheter-days are reported in critically ill children $(33,34)$. This institution does not screen for catheter-related-thromboses, so asymptomatic thrombosis was not evaluated. The four additional 'silent' venous occlusions noted above were found incidentally during subsequent HD-CVC placement. The overall rate of thromboses/silent occlusions is high, but not surprising given the patients' small size. Chylothorax is an additional concern, especially with upper venous system HD-CVCs, where resistance to lymphatic drainage arises from obstruction by the CVC, or from venous thrombosis (35-37). Chylothorax may cause serious morbidity, prolonged ICU and hospital stays and death, as occurred here $(35,38)$.

The overall CRBSI rate of 1.03 infections/1,000 catheter-days (tunneled and non-tunneled catheters) is within reported rates of $0.3-10$ infections/1,000 catheter-days in infants with tunneled HD-CVC (4, 5, 8-11). The significantly higher rates among non-tunneled HDCVCs are multifactorial in origin. Of the 5 CRBSI, only one was present at the time of the patient's death. 
Achieving and maintaining RA catheter tip position in infants is challenging. In adults, the 'functional tip' (distal side hole to catheter tip) is recommended to be within the RA without touching the atrial floor (14). To achieve this in infants with current catheter designs is difficult as catheter distances between staggered ends are frequently too long. Trimming the catheter tip is 'off-label', but alternative options are limited. Trimming potentially increases rate of dialysis recirculation and roughens the tip, promoting thromboses. However, no significant association between trimmed catheters and fibrin sheath/thromboses was found. For tunneled catheters, gauging tunnel length in an infant's lax, mobile subcutaneous tissues is difficult. The catheter can adopt a short tight curve or a wide long course in the tunnel (causing a low or high tip position). If the former occurs, catheter exchange by externalization at the neck and over-the-wire-exchange through a new longer tunnel is associated with blood loss and difficulties in infants with little catheter length/purchase. Extending the tunnel was deemed less invasive and avoided entire catheter replacement. Maintaining RA position was also impacted by infant growth, resolution of body edema, changes in thoracic/abdominal size with nephrectomies/transplants, catheter dislodgement by patients, and migration following loss of sutures. Non-tunneled HD-CVCs frequently are too long for infants' thorax, but are not suitable for trimming given their tapered tips. Suturing them high to the scalp is uncomfortable for the patient and difficult for nursing/dialysis care. Other options include use of "soft" non-tunneled catheters that can be curved and sutured to infant's thorax, used of cuffed catheters as non-tunneled lines with cuff exposed, or use of symmetric tip catheters that may obviate the need for trimming. These challenges highlight a need and opportunity for manufacturers to modify product design. 
Non-tunneled HD-CVCs rarely required maintenance procedures, compared to $74 \%$ of tunneled. However, the difference per 1,000 catheter days was not significant. Quinlan et al similarly found that $67 \%$ of children $<10 \mathrm{~kg}$ required line revisions (9). Non-tunneled HD-CVC had significantly higher infection and thrombosis rates compared to tunneled HD-CVC. Tunneled HD-CVCs are therefore favored at this institution, even if dialysis duration is uncertain. Nonetheless, non-tunneled HD-CVCs may be needed in unstable critically ill infants, obviating general anesthesia, longer procedure times, and cardiac irritability.

Study limitations include retrospective design and small sample size, making multivariate analyses not possible. Although catheters placed by other services were excluded, IR places most HD-CVCs in this institution. The number of catheter dysfunctions may be underestimated, as every pressure alarm, flow reversal and tissue plasminogen activator use was not individually analyzed (35). However, most were captured as institutional practice is to have persistent/recurring alarms evaluated by IR. Primary device service intervals may similarly be impacted. Two patients who recovered were lost to follow up after discharge a potential source of bias. Attributing thromboses to a specific CVC is difficult when patients had other CVCs. Incidence of venous thrombosis/occlusions diagnosed by US or during HD-CVC insertions is likely an underestimate. Retrospective confirmation that all infections met every criterion (e.g. $>2$ hour differential between central and peripheral positive cultures) was not possible (39). However, removal of life-saving HD-CVCs were 
only undertaken when absolutely necessary and without other sources of infection identified.

\section{Conclusion}

In infants HD-CVCs insertion and maintenance is challenging. Technical modifications are required. Multiple dialysis cycles and IR-maintenance procedures are expected. Nontunneled HD-CVCs are associated with higher CRBSI and thrombosis rates. Vascular occlusions/thromboses are frequent. Health care providers can advise parents of these challenges when embarking on RRT in infancy. 


\section{References}

1. Rees L (2002) Management of the infant with end-stage renal failure. Nephrol Dial Transplant 17:1564-1567.

2. Jalanko H, Mattila I, Holmberg C (2016) Renal transplantation in infants. Pediatr Nephrol 31:725-735.

3. Salvatierra O, Jr., Millan M, Concepcion W (2006) Pediatric renal transplantation with considerations for successful outcomes. Semin Pediatr Surg 15:208-217.

4. Al-Hermi BE, Al-Saran K, Secker D, Geary DF (1999) Hemodialysis for end-stage renal disease in children weighing less than $10 \mathrm{~kg}$. Pediatr Nephrol 13:401-403.

5. Feinstein S, Rinat C, Becker-Cohen R, Ben-Shalom E, Schwartz SB, Frishberg Y

(2008) The outcome of chronic dialysis in infants and toddlers--advantages and drawbacks of haemodialysis. Nephrol Dial Transplant 23:1336-1345.

6. Alexander RT, Foster BJ, Tonelli MA, Soo A, Nettel-Aguirre A, Hemmelgarn BR, Samuel SM (2012) Survival and transplantation outcomes of children less than 2 years of age with end-stage renal disease. Pediatr Nephrol 27:1975-1983.

7. Hijazi R, Abitbol CL, Chandar J, Seeherunvong W, Freundlich M, Zilleruelo G (2009) Twenty-five years of infant dialysis: a single center experience. J Pediatr 155:111117.

8. Pollack S, Eisenstein I, Tarabeih M, Shasha-Lavski H, Magen D, Zelikovic I (2016) Long-term hemodialysis therapy in neonates and infants with end-stage renal disease: a 16year experience and outcome. Pediatr Nephrol 31:305-313.

9. Quinlan C, Bates M, Sheils A, Dolan N, Riordan M, Awan A (2013) Chronic hemodialysis in children weighing less than $10 \mathrm{~kg}$. Pediatr Nephrol 28:803-809.

10. Shroff R, Wright E, Ledermann S, Hutchinson C, Rees L (2003) Chronic hemodialysis in infants and children under 2 years of age. Pediatr Nephrol 18:378-383.

11. Kovalski Y, Cleper R, Krause I, Davidovits M (2007) Hemodialysis in children weighing less than $15 \mathrm{~kg}$ : a single-center experience. Pediatr Nephrol 22:2105-2110. 12. Paglialonga F, Consolo S, Pecoraro C, Vidal E, Gianoglio B, Puteo F, Picca S, Saravo MT, Edefonti A, Verrina E (2016) Chronic haemodialysis in small children: a retrospective study of the Italian Pediatric Dialysis Registry. Pediatr Nephrol 31:833-841.

13. Chait PG, Temple M, Connolly B, John P, Restrepo R, Amaral JG (2002) Pediatric interventional venous access. Tech Vasc Interv Radiol 5:95-102.

14. Tal M, Friedman T, Mojibian H Dialysis catheter tip placement: the functional tip. Enovascular Today 2013:73-75.

15. Santoro D, Benedetto F, Mondello P, Pipito N, Barilla D, Spinelli F, Ricciardi CA, Cernaro V, Buemi M (2014) Vascular access for hemodialysis: current perspectives. Int J Nephrol Renovasc Dis 7:281-294.

16. Silberzweig JE, Sacks D, Khorsandi AS, Bakal CW, Society of Interventional Radiology Technology Assessment C (2003) Reporting standards for central venous access. J Vasc Interv Radiol 14:S443-452.

17. Adeb M, Baskin KM, Keller MS, Krishnamurthy G, Nijs E, Meyers K, Pradhan M, Cahill AM (2012) Radiologically placed tunneled hemodialysis catheters: a single pediatric institutional experience of 120 patients. J Vasc Interv Radiol 23:604-612.

18. Miller DL, O'Grady NP, Society of Interventional R (2012) Guidelines for the prevention of intravascular catheter-related infections: recommendations relevant to 
interventional radiology for venous catheter placement and maintenance. J Vasc Interv Radiol 23:997-1007.

19. Baskin KM, Mermel LA, Saad TF, Journeycake JM, Schaefer CM, Modi BP, Vrazas JI, Gore B, Drews BB, Doellman D, Kocoshis SA, Abu-Elmagd KM, Towbin RB, Venous Access: National G, Registry Development Initiative Affected Persons Advisory P (2019) Evidence-Based Strategies and Recommendations for Preservation of Central Venous Access in Children. JPEN J Parenter Enteral Nutr 43:591-614.

20. Khalilzadeh O, Baerlocher MO, Shyn PB, Connolly BL, Devane AM, Morris CS, Cohen AM, Midia M, Thornton RH, Gross K, Caplin DM, Aeron G, Misra S, Patel NH, Walker TG, Martinez-Salazar G, Silberzweig JE, Nikolic B (2017) Proposal of a New Adverse Event Classification by the Society of Interventional Radiology Standards of Practice Committee. J Vasc Interv Radiol 28:1432-1437 e1433.

21. Vo JN, Hoffer FA, Shaw DW (2010) Techniques in vascular and interventional radiology: pediatric central venous access. Tech Vasc Interv Radiol 13:250-257.

22. Allon M, Brouwer-Maier DJ, Abreo K, Baskin KM, Bregel K, Chand DH, Easom AM, Mermel L, Mokrzycki MH, Patel PR, Roy-Chaudhury P, Shenoy S, Valentini RP, Wasse H (2018) Recommended Clinical Trial End Points for Dialysis Catheters. Clin J Am Soc Nephrol 13:495-500.

23. Rees L (2014) The dilemmas surrounding the decision to start chronic dialysis in the neonate. Kidney Int 86:18-20.

24. Bunchman TE (1995) Chronic dialysis in the infant less than 1 year of age. Pediatr Nephrol 9 Suppl:S18-22.

25. Bunchman TE (1996) The ethics of infant dialysis. Perit Dial Int 16 Suppl 1:S505508.

26. Carey WA, Talley LI, Sehring SA, Jaskula JM, Mathias RS (2007) Outcomes of dialysis initiated during the neonatal period for treatment of end-stage renal disease: a North American Pediatric Renal Trials and Collaborative Studies special analysis. Pediatrics 119:e468-473.

27. van Stralen KJ, Borzych-Duzalka D, Hataya H, Kennedy SE, Jager KJ, Verrina E, Inward C, Ronnholm K, Vondrak K, Warady BA, Zurowska AM, Schaefer F, Cochat P, registry EE-E, registry I, registry A, Japanese RRTr (2014) Survival and clinical outcomes of children starting renal replacement therapy in the neonatal period. Kidney Int 86:168174.

28. Rees L (2017) Renal replacement therapies in neonates: issues and ethics. Semin Fetal Neonatal Med 22:104-108.

29. Vidal E, Edefonti A, Murer L, Gianoglio B, Maringhini S, Pecoraro C, Sorino P, Leozappa G, Lavoratti G, Ratsch IM, Chimenz R, Verrina E, Italian Registry of Paediatric Chronic D (2012) Peritoneal dialysis in infants: the experience of the Italian Registry of Paediatric Chronic Dialysis. Nephrol Dial Transplant 27:388-395.

30. Zurowska AM, Fischbach M, Watson AR, Edefonti A, Stefanidis CJ, European Paediatric Dialysis Working G (2013) Clinical practice recommendations for the care of infants with stage 5 chronic kidney disease (CKD5). Pediatr Nephrol 28:1739-1748.

31. Jha V, Martin DE, Bargman JM, Davies S, Feehally J, Finkelstein F, Harris D, Misra M, Remuzzi G, Levin A (2017) Ethical issues in dialysis therapy. Lancet 389:18511856.

32. Kahrass H, Strech D, Mertz M (2016) The Full Spectrum of Clinical Ethical Issues in Kidney Failure. Findings of a Systematic Qualitative Review. PLoS One 11:e0149357. 
33. Smitherman AB, Alexander T, Connelly M, Snavely AC, Weston BW, Liles EA, Steiner MJ (2015) The incidence of catheter-associated venous thrombosis in noncritically ill children. Hosp Pediatr 5:59-66.

34. Faustino EV, Spinella PC, Li S, Pinto MG, Stoltz P, Tala J, Card ME, Northrup V, Baker KE, Goodman TR, Chen L, Silva CT (2013) Incidence and acute complications of asymptomatic central venous catheter-related deep venous thrombosis in critically ill children. J Pediatr 162:387-391.

35. Borasino S, Diaz F, El Masri K, Dabal RJ, Alten JA (2014) Central venous lines are a risk factor for chylothorax in infants after cardiac surgery. World J Pediatr Congenit Heart Surg 5:522-526.

36. Anton N, Cox PN, Massicotte MP, Chait P, Yasui Y, Dinyari PM, Marzinotto V, Mitchell LG (2009) Heparin-bonded central venous catheters do not reduce thrombosis in infants with congenital heart disease: a blinded randomized, controlled trial. Pediatrics 123:e453-458.

37. Hanslik A, Thom K, Haumer M, Kitzmuller E, Albinni S, Wolfsberger M, SalzerMuhar U, Male C (2008) Incidence and diagnosis of thrombosis in children with short-term central venous lines of the upper venous system. Pediatrics 122:1284-1291.

38. Carter S, van de Hoef D, Temple M, Harvey E, Al-Saleh S, Licht C, Noone D (2017) Plastic bronchitis: a rare complication of long-term haemodialysis catheter placement in a child. Pediatr Nephrol 32:1635-1638.

39. Mermel LA, Allon M, Bouza E, Craven DE, Flynn P, O'Grady NP, Raad, II, Rijnders BJ, Sherertz RJ, Warren DK (2009) Clinical practice guidelines for the diagnosis and management of intravascular catheter-related infection: 2009 Update by the Infectious Diseases Society of America. Clin Infect Dis 49:1-45. 


\section{Figure Legends}

Fig. 122 day old male, $3.1 \mathrm{~kg}$, with nephrotic syndrome and ESRD requiring hemodialysis. An 8 French, 15cm cuff-to-tip, Medcomp HD-CVC was inserted after trimming the catheter's proximal lumen (venous return). Note the location of the catheter hub (*) and overall line length with respect to the patient's chest size (A). Note the distance between both lumens ([) and its position in the right atrium despite trimming the catheter (B). The atriocaval junction (arrow) is estimated at 2 vertebrae below the carina, around T6. 